\title{
Effects of Azelnidipine-Carboxymethylcellulose Gel on Healing of Full-Thickness Skin Wounds in Streptozotocin Induced Diabetic Rats
}

This article was published in the following Dove Press journal: Veterinary Medicine: Research and Reports

\author{
Mohammad Yasin Karami $\mathbb{D}^{1,2}$ \\ Nasrin Mansournia' \\ Neda Bagherian ${ }^{3}$ \\ Alireza Makarem (iD) 4 \\ Nader MoeinVaziri ${ }^{5}$ \\ Sahar Borna ${ }^{6}$ \\ Amir Hossein Pourdavood ${ }^{7}$ \\ Iman Shamohammadi ${ }^{4}$ \\ 'Metabolic Disease Research Center, \\ Faculty of Medicine, AJA University of \\ Medical Sciences, Tehran, Iran; ${ }^{2}$ Breast \\ Disease Research Center, Faculty of \\ Medicine, Shiraz University of Medical \\ Sciences, Shiraz, Iran; ${ }^{3}$ Department of \\ Pediatrics, Faculty of Medicine, Shiraz \\ University of Medical Sciences, Shiraz, \\ Iran; ${ }^{4}$ Department of Urology, Faculty of \\ Medicine, Shiraz University of Medical \\ Sciences, Shiraz, Iran; ${ }^{5}$ Department of \\ Surgery, Faculty of Medicine, Shiraz \\ University of Medical Sciences, Shiraz, \\ Iran; 'Student Research Committee, \\ Faculty of Medicine, Sari University of \\ Medical Sciences, Sari, Iran; ${ }^{7}$ Department \\ of Surgery, Faculty of Medicine, Kerman \\ University of Medical Sciences, Kerman, \\ Iran
}

Correspondence: Neda Bagherian Department of Pediatrics, Faculty of Medicine, Shiraz University of Medical

Sciences, Shiraz, Iran

Tel +989177021044

Email bagheriannn@gmail.com
Objective: To evaluate the effects of azelnidipine-carboxyl methyl cellulose (AZL-CMC) gel and carboxyl methyl cellulose $2 \%$ gel (CMC) on the healing of full-thickness skin wounds of diabetic rats.

Methods: Fifteen Sprague Dawley male rats were studied. The rats were divided into three groups: AZL-CMC gel-treated, CMC 2\% gel-treated, and control group. Wounds were assessed by wound area measurement every 3 days and histopathology samples were collected at 4, 7 and 12 days post wounding to evaluate the healing process using stereological study. Mann-Whitney $U$-test repeated measurement and non-parametric one-way analysis of variance (ANOVA) were used to analyze the data using SPSS, version 18.

Results: Numerical density of the fibroblasts of the AZL-CMC gel treated group was 59.17 $\pm 2.69\left(\times 10^{4} / \mathrm{mm}^{3}\right)$ and higher than the control $22.64 \pm 1.34\left(\times 10^{4} / \mathrm{mm}^{3}\right)$ and CMC $2 \%$-treated groups $40.80 \pm 5.27\left(\times 10^{4} / \mathrm{mm}^{3}\right)$, respectively, $\mathrm{P}<0.001$. The volume density of the collagen bundles and LV of the vessels were $83.1 \pm 4.46$ and $42.16 \pm 5.78$, respectively, in the AZLCMC treated group, and higher compared to the control $(53.96 \pm 5.07,9.9 \pm 2.49)$ and the CMC $2 \%$-treated $(65.88 \pm 2.13,18.1 \pm 2.20)$ groups $(\mathrm{P}<0.001)$.

Conclusion: The healing of AZL-CMC gel-treated wound was better than the control wounds, grossly. Wound healing processes and wound closure in the intervention group began sooner and was completed more quickly. The quantitative and qualitative parameters showed the significant wound healing effect of the AZL-CMC gel-treated group.

Keywords: wound healing, azelnidipine, streptozotocin, diabetic, rat

\section{Introduction}

Diabetic foot ulcers (DFU) are the most common wounds in diabetic patients. ${ }^{1}$ The likelihood of a diabetic person to have foot ulcers throughout his life is $15-25 \%{ }^{2}$ Approximately, 50-70\% of all the limb amputations are due to diabetic wounds and it has been reported that in every $30 \mathrm{~s}$, one leg is amputated due to diabetic wound worldwide. $^{1}$

Full-thickness wounds are described with complete destruction of the epidermis and dermis in addition to deeper structures. ${ }^{3}$ High levels of blood glucose can damage the nerve fiber through various mechanisms, including increased levels of reactive oxygen species and their unwanted effects, like the activation of redoxsensitive transcription factors, ${ }^{4}$ nitric oxide blockage, and increased concentrations of the matrix metalloproteinase. Moreover, vascular disease and ischemia usually reduce the healing capacity. ${ }^{5-7}$ 
Some studies have shown that Calcium Channel Blockers (CCBs) have beneficial effects in other conditions than HTN (Hypertension), such as wound healing, and in animal model studiesdiltiazem, verapamil, nifedipine, and azelnidipine have been proposed to play potential roles. ${ }^{8,9}$

Evidence has shown that $\mathrm{CCBs}$ have antioxidant properties which increase collagen accumulation and fibroblast proliferation by promotion of nitric oxide (NO) production; they are known to have vasodilatory properties, increasing blood flow to the wound area and stimulating the production of the growth factor. The improvement of insulin sensitivity by CCB in various diabetic models has been reported. ${ }^{10,11}$

Keratinocytes functionally express several types of calcium channels; ${ }^{12}$ also, tissue collagenases, such as matrix metalloproteinase, are expressed more abundantly at tissue injury sites and their action depends on the associated inflammatory cytokines and intracellular calcium levels. ${ }^{11}$ By acting on the voltage gated $\mathrm{Ca} 2+$ channels, nifedipine and amlodipine altered intracellular calcium and had antioxidant activity in some in vitro studies. ${ }^{13}$ Further azelnidipine accelerates wound healing, improves NO levels in the wound fluid, and increases the density of the collagen fibers, numerical density of the fibroblasts, and length density of the vessels. ${ }^{7}$

Azelnidipine could reduce the expression of the subunits of NADPH oxidase, a key class of enzymes in superoxide production, or NADPH oxidase-mediated ROS generation, and it may be possible that the improvement of insulin resistance by azelnidipine is caused by the change in the muscle blood flow. ${ }^{10}$

Extracellular calcium contents and calcium influx into the keratinocytes also regulate the barrier recovery ${ }^{12}$ and the restoration of the barrier function in order to prevent further damage, or infection seems to be one of the main reasons for skin wound healing. ${ }^{3}$

In addition to growth factors, stem cells, nanoparticles, platelet-rich plasma and other wound treatments, ${ }^{14}$ wound healing treatment requires the use of a wide range of dressing materials: films, foams, hydrogels, hydrocolloids, and impregnated coverings containing antimicrobial agents, enzymes, and various biologically active substances. ${ }^{15,16}$ Hydrogels take advantage of a hydrophilic material that absorbs a certain amount of exudate but keeps a moist environment. ${ }^{17}$

Carboxymethyl cellulose (CMC) is a water-soluble semisynthetic, ether derivative of cellulose which can be crosslinked to make biodegradable hydrogel, and it has been used in drug carriers for wound care due to its ability to absorb a significant amount of water and good biocompatibility. ${ }^{18,19}$

We, therefore, aimed to assess the efficacy of treatment with a combination of azelnidipine and CMC gel dressing to improve the wound closure, with macroscopic and microscopic aspects of cutaneous wound healing in an experimental diabetes model.

\section{Materials and Methods}

\section{Experimental and Diabetic Animals}

Thirty male 2-month-old Sprague-Dawley rats weighting 200-250 g were selected for this study. The animals were kept in controlled temperature condition $(25 \mathrm{C})$ with $12 \mathrm{~h}$ light-dark cycles for at least 6 days before use and free access to water and food (except for the times of fasting necessary for biochemical assays). The present study was in accordance with the standards of the National Society of Medical Research ("Principles of Laboratory Animal Care") and "The Guide for the Care and Use of Laboratory Animals" (NIH publication No. 86-23, revised 1985), and the ARRIVE guideline for reporting animal research. ${ }^{20}$ The rats were free of all pathogens listed in the FELASA (Federation of European Laboratory Animal Science Associations) recommendations ${ }^{21}$ and the study was approved by the Ethics Committee of AJA University of Medical Sciences. Tehran, Iran (No: IR. AJAUMS.MED.REC.1393).

After $12 \mathrm{hrs}$ of fasting, 30 rats received a single dose (60 $\mathrm{mg} / \mathrm{kg}$ ) of intra-peritoneal Streptozotocin (Sigma, St. Louis, MO, USA) dissolved in sterile citrate buffer $(0.05 \mathrm{~mol} / \mathrm{L}$ sodium citrate, $\mathrm{pH}$ 4.5). Five days later, after an overnight fasting, $1 \mathrm{~mL}$ blood sample was taken from each rat's tail vein. The samples were centrifuged, and plasma glucose levels measured by a glucose analyzer (Easy GlucoInfopia Co., Ltd., Anyang, Gyeonggi-do, Korea). The rats with plasma glucose level higher than $280 \mathrm{mg} / \mathrm{dL}$ were considered to be diabetic ${ }^{22}$ and used for the next steps of the experiment. Fifteen non-diabetic rats were excluded and fifteen rats were included randomly in the study groups. The rats were divided into three groups: AZL-CMC Gel-treated (experimental group), CMC 2\% Gel-treated (Positive Placebo) and Control group (Negative Placebo). We used only male rats to reduce female rat hormonal effect bias on wound healing.

\section{Preparation of AZL-CMC Gel and CMC $2 \% \mathrm{Gel}$}

AZL-CMC Gel was supplied by MNPCRC (Medicinal and Natural Products Chemistry Research Center, AJA 
University of Medical Sciences, Tehran, Iran). In order to facilitate the application of the agent, we provided $4 \%$ AZL-CMC Gel by dissolving 4g AZL in 2cc ethanol ( $70 \%)$ and then transferring the solution into $2 \%$ carboxymethylcellulose (CMC) ( $2 \mathrm{~g} \mathrm{CMC}$ dissolved in $98 \mathrm{cc}$ distilled water). The gel base (vehicle) was also supplied by creating $2 \%$ CMC gel without the AZL component.

\section{Wound Healing Activity}

The dorsum of the waist of the Sprague Dawley rats' skin was shaved before starting the excision wound healing experiments. Animals were anesthetized with intra-peritoneal Ketamine (sigma Aldrich, United States of America, $80 \mathrm{mg} / \mathrm{kg}$ ) and Xylazine (sigma Aldrich, United States of America, $3 \mathrm{mg} / \mathrm{kg}$ ).

One full-thickness excisional wound (circular area of about $150 \mathrm{~mm}^{2}$ and $2 \mathrm{~mm}$ depth) was created along with the markings about $14 \mathrm{~mm}$ in diameter. All wounds were photographed during the wound healing period on the days of operation, 3, 6, 9 and 12 .

The wound area was calculated using Adobe Photoshop CS software (version 5). Electrocardiogram (ECG) paper was used for reference scale. ${ }^{23}$ The unhealed area (\%) was calculated as:

unhealed area,,$(\%)=[($ area at visit $1-$ area at each visit $) /$ (area at visit 1$)] \times 100$

\section{Tissue Preparation and Processing}

Incisional biopsy $(4 \times 4 \mathrm{~mm})$ of the wounds and normal skin was performed in 6 O'clock area of wounds on the 4th day and 12 O'clock area of wounds on the 7th day for histopathologic study. The rats were euthanized by ether overdose 12 days after wounding, respectively. The end of the study was set according to a prior pilot study (day 12). After day 12, full thickness skin biopsies $(1.5 \times 1.5 \mathrm{~cm})$ were taken from the epithelialized wound sites and fixed in buffered formaldehyde $(\mathrm{pH}=7.2)$, for histopathological analysis.

Nine pieces were chosen for stereological analysis. The pieces were embedded in a cylindrical paraffin block to obtain isotropic systematic random sections. The volume density of the collagen fibers, length density of the vessels, and numerical density of the fibroblasts were studied by stereological methods. ${ }^{24}$

Also, the samples were stained with hematoxylin and eosin (H\&E) and Masson's trichrome method at a magnification of $\times 100-400$. The authors used the Abramov's histological scoring system and Stacie Scardno's study.23,25 The study was blinded to the histologist who performed the histopathological review.

\section{Statistical Analysis}

The results are expressed as mean \pm standard deviation (SD) in the text and figures. The relative wound area and histopathological analysis were performed using the nonparametric test, repeated measurement one-way analysis of variance (ANOVA) and Mann-Whitney $U$-test. Differences were considered statistically significant in the case of $\mathrm{P}<0.05$. Data were analyzed using statistical software SPSS 18.0 for windows.

\section{Results}

\section{Area of the Wounds}

The differences between the three groups regarding the primary wound areas were not significant. The rate of wound closure in the AZL-CMC Gel treated group was significantly higher in comparison to the control and CMC $2 \%$ treated groups $(\mathrm{P}<0.05)$. Also, the rate of wound closure in the CMC $2 \%$ treated wounds was higher than the control group wounds $(\mathrm{P}<0.05)$. (Table 1, Figure 1).

\section{Histologic Results Fibroblast Population}

Numerical density of the fibroblasts $(\mathrm{Nv})$ in the dermis of the AZL-CMC Gel treated group was significantly higher than that of the control and CMC $2 \%$ treated groups. Mean \pm SD of numerical density of the fibroblasts in the AZLCMC-treated groups was reported as $59.17 \pm 2.69\left(\times 10^{4} /\right.$ $\left.\mathrm{mm}^{3}\right)$ and higher than the controls $\left(22.64 \pm 1.34\left(\times 10^{4} /\right.\right.$ $\left.\left.\mathrm{mm}^{3}\right), \quad \mathrm{P}<0.001\right)$ and $\mathrm{CMC} 2 \%$-treated group. (40.80 $\left.\pm 5.27\left(\times 10^{4} / \mathrm{mm}^{3}\right), \mathrm{P}<0.001\right)$ (Table 2).

\section{Volume Density of the Collagen Bundles}

The Mean \pm SD of the volume density of the collagen bundles (\%) was $83.1 \pm 4.46$ in the AZL-CMC treated group and higher compared to the control $(53.96 \pm 5.07)$ and the CMC 2\%-treated (65.88 \pm 2.13$)$ groups, which were both statistically significant $(\mathrm{P}<0.001)$ (Table 2$)$.

\section{Length Density of the Vessels}

$\mathrm{LV}$ of the vessels was significantly increased in the wound tissues of AZL-CMC-treated compared with CMC $2 \%$ treated and control groups $(\mathrm{P}<0.001)$ (Table 2$)$. 
Table I Wound Surface Area in Diabetic Rats

\begin{tabular}{|c|c|c|c|c|}
\hline \multirow[t]{4}{*}{ Day } & \multicolumn{3}{|c|}{ Mean \pm SD $\left(\mathrm{mm}^{2}\right)$} & \multirow{2}{*}{$\begin{array}{l}\text { P-Value } \\
\text { CMC/Control }\end{array}$} \\
\hline & AZL-CMC Gel & CMC & Control & \\
\hline & & & & AZL-CMC/Control \\
\hline & & & & CMC/AZL-CMC \\
\hline 0 & $149.4 \pm 10.55$ & $148.80 \pm 8.49$ & $149.4 \pm 7.4$ & $\begin{array}{l}P>0.05 \\
P>0.05 \\
P>0.05\end{array}$ \\
\hline 3 & $102.8 \pm 11.21$ & $128.40 \pm 5.12$ & $136.00 \pm 1.58$ & $\begin{array}{l}P=0.013 \\
P<0.001 \\
P<0.002\end{array}$ \\
\hline 6 & $39.00 \pm 8.51$ & $104.80 \pm 2.49$ & $118.00 \pm 4.12$ & $\begin{array}{l}P<0.001 \\
P<0.001 \\
P<0.001\end{array}$ \\
\hline 9 & $9.20 \pm 2.58$ & $77.00 \pm 7.17$ & $95.20 \pm 6.64$ & $\begin{array}{l}P<0.003 \\
P<0.001 \\
P<0.001\end{array}$ \\
\hline 12 & $0.01 \pm 0.001$ & $45.2 \pm 6.35$ & $75.4 \pm 7.95$ & $\begin{array}{l}P<0.001 \\
P<0.001 \\
P<0.001\end{array}$ \\
\hline
\end{tabular}

\section{Histopathology Results}

Histopathology results according to Abramov's histological scoring system and Scardno's study showed antiinflammatory, anti-hemorrhagic and anti-necrotic and

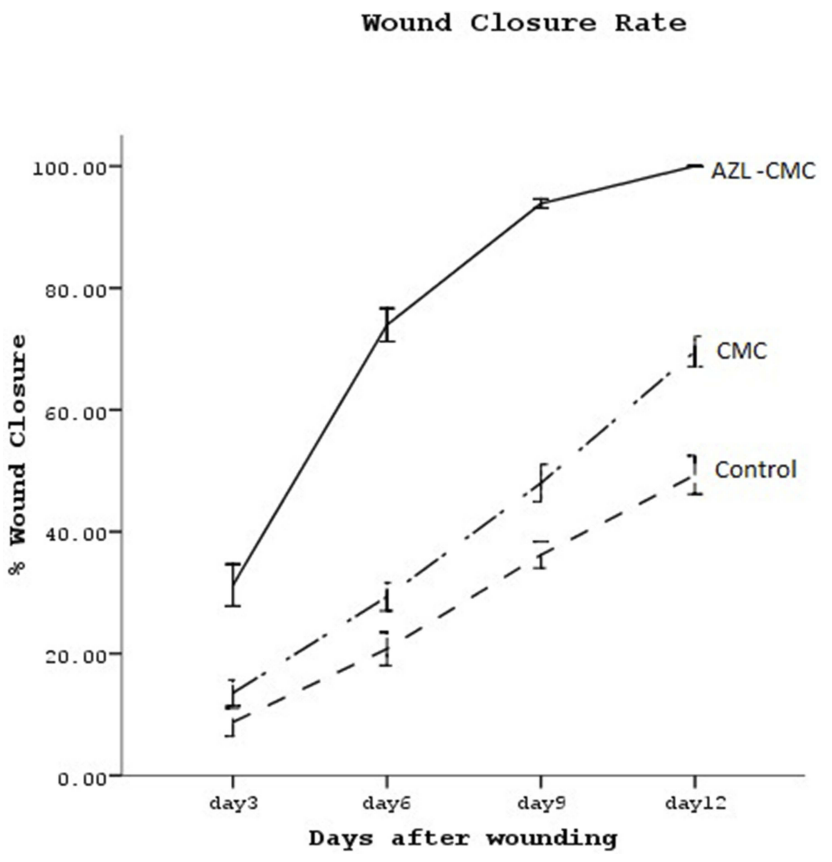

Figure I Wound closure rate. neovascularization, collagen fiber arrangement effects of AZL-CMC gel-treated better than the control group which lead to early healing of skin wounds in diabetic rats. (Figures 2 and 3).

\section{Discussion}

The present study demonstrated that Azelnidipine increased the wound healing speed in diabetic rats, and the rate of wound closure, numerical density of the fibroblasts, volume density of the collagen bundles and LV of vessels in AZLCMC Gel treated group were significantly higher in comparison to the control and CMC $2 \%$ treated groups. We also studied and described the histopathologic characteristic of the AZL-CMC Gel treated wounds by using Abramov's histological scoring system.

Our study histopathology results showed anti-inflammatory, anti-hemorrhagic and anti-necrotic effects of AZL-CMC gel which can be one of the most important characteristics of wound treatment.

It has been reported that $\mathrm{CCBs}$ can attenuate the oxidative stress, and azelnidipine further improves the glucose intolerance and glucose uptake and reduces in situ superoxide production in the skeletal muscle. Azelnidipine can reduce the expression of the subunits of NADPH oxidase, a key enzyme in superoxide production, or NADPH oxidase-mediated ROS 
Table 2 Effect of AZL-CMC Gel on Wound Healing in Stereological Study. Control, CMC Treated and AZL-CMC-Treated Wounded Rats

\begin{tabular}{|c|c|c|c|c|}
\hline \multirow[t]{4}{*}{ Wound } & \multirow[t]{4}{*}{ AZL-CMC Gel } & \multirow[t]{4}{*}{ CMC $2 \%$ Gel } & \multirow[t]{4}{*}{ Control } & P-Value \\
\hline & & & & AZL-CMC/Control \\
\hline & & & & CMC/Control \\
\hline & & & & AZL-CMC/CMC \\
\hline $\mathrm{NV}$ of Fibroblast $\left(\times 10^{4} / \mathrm{mm}^{3}\right)$ & $59.17 \pm 2.69$ & $40.80 \pm 5.27$ & $22.64 \pm 1.34$ & $\begin{array}{l}P<0.001 \\
P<0.001 \\
P<0.001\end{array}$ \\
\hline Vv of Collagen Fiber (collagen/dermis) (\%) & $83.1 \pm 4.46$ & $65.88 \pm 2.13$ & $53.96 \pm 5.07$ & $\begin{array}{l}P<0.001 \\
P<0.01 \\
P<0.001\end{array}$ \\
\hline $\mathrm{LV}$ of vessels $\left(\mathrm{mm} / \mathrm{mm}^{3}\right)$ & $42.16 \pm 5.78$ & $18.1 \pm 2.20$ & $9.9 \pm 2.49$ & $\begin{array}{l}P<0.001 \\
P<0.01 \\
P<0.001\end{array}$ \\
\hline
\end{tabular}

generation. ${ }^{26,27}$ This is why we chose AZL to examine our hypothesis about the wound healing capacity of this calcium channel blocker.

However, azelnidipine modulates the intracellular signaling of insulin and Angiotensin II by blockage of calcium channel. These intracellular actions of AZL seem to be, at least partly, mediated by the inhibition of oxidative stress.

Probably, the improvement of insulin resistance by azelnidipine is caused by the change in the muscle blood flow. As we showed in this study, the LV of the vessels was significantly increased in the wound tissue of the AZLCMC-treated group which can also explain the insulin resistance reduction beside the other functions of AZL.

AZL increases the wound fluid nitrite level to the values similar to those in non-DM animals. However, AZL does not change the decreased plasma NO level in diabetic rats. ${ }^{9}$

Previous studies have demonstrated the importance of nitric oxide for successful wound healing. In diabetes, NO synthesis reduces in the wound. ${ }^{28}$ Bagheri et al. studied 44 male rats and hypothesized that azelnidipine (AZL) with antioxidant properties would enhance wound healing in streptozotocin-induced diabetic rats by restoring $\mathrm{NO}$ synthesis. Similar to our article, they evaluated the wound area and wound closure rate. The volume density of the collagen fibers, numerical density of the fibroblasts, length density of the vessels and plasma and wound fluid nitrite were estimated. They showed AZL accelerated the wound healing rate and also improved the wound fluid NO level toward normal value in diabetic rats. The volume density of the collagen fibers, numerical density of the fibroblasts, and length density of the vessels were increased in the AZL-treated rats compared with the control group. Compared with the control group, wound fluid nitrite level was significantly increased in the AZL-treated rats. ${ }^{9}$ We did not measure the wound fluid NO level, but we focused on the histopathology results analysis and AZL anti-inflammatory action.

Wound dressings have to be multifunctional: to facilitate debridement, stimulate the regeneration of healthy tissue, prevent bacterial contamination, and at the same time maintain a moist environment and prevent the formation of contractures. ${ }^{15,18}$ We used AZL-CMC Gel, which is a synthetic mediator, with an underlying gelatinous substance (CMC 2\%).

Zekavat et al. ${ }^{23}$ evaluated hydroalcoholic extract-based carboxy methyl cellulose (CMC) gel of Punica granatum peel (PCMC) and CMC effects on the healing of fullthickness skin wounds, which was also investigated in our study. Forty-two rats were studied. The results showed that wound healing took significantly longer in the wounds treated with normal saline than those treated with PCMC and $\mathrm{CMC}$ gel; also, wound healing took a significantly shorter time in the wounds treated with CMC than those treated with $\mathrm{PCMC}$ in both diabetic and nondiabetic rats, and as we had the same outcome by using the CMC hydrogel, the rate of wound closure in the group treated with the CMC 2\% was higher than the control group.

In the same line, Ashkani Esfahani et al.'s study investigated the effects of verapamil on the wound healing 

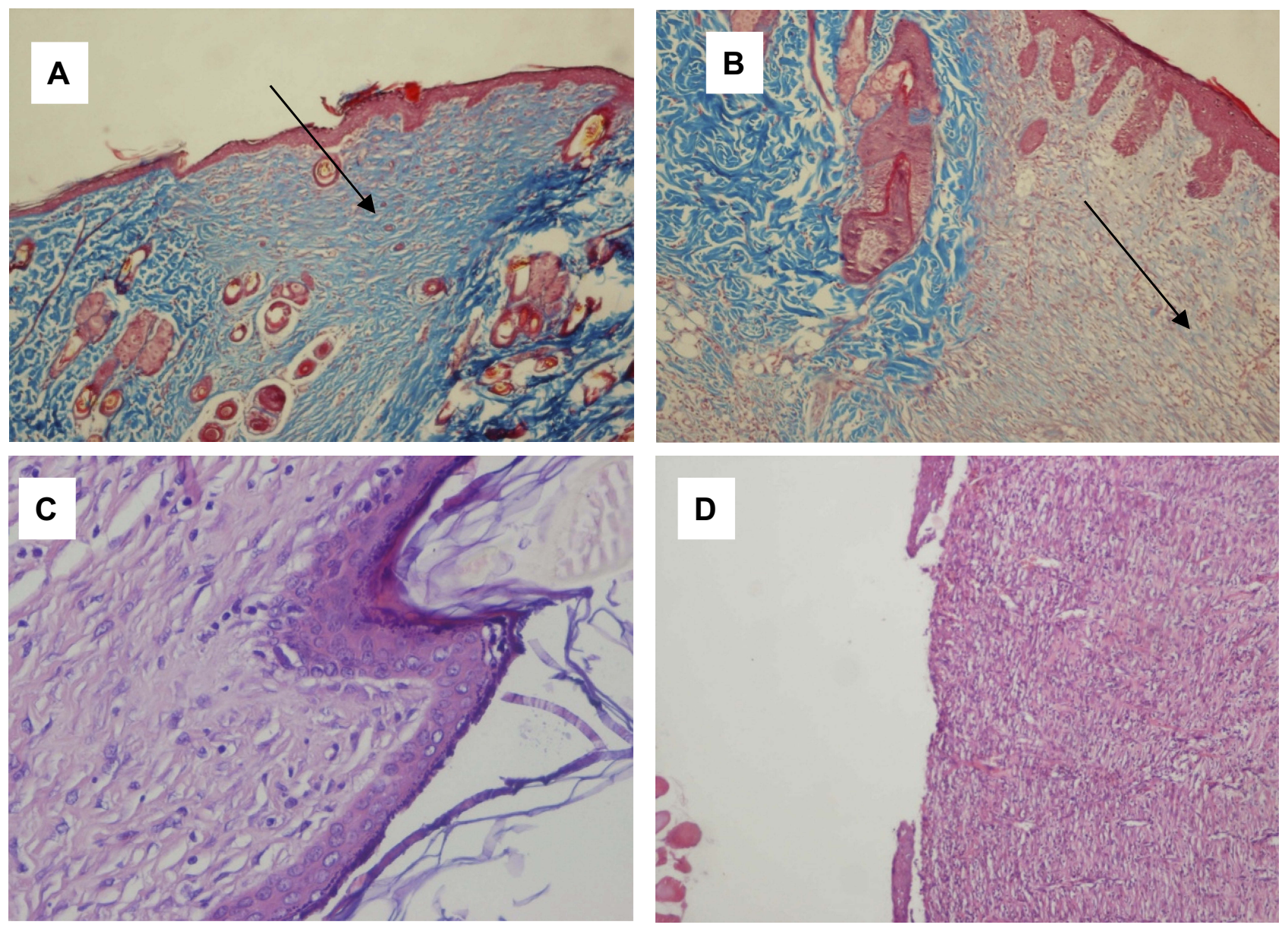

Figure 2 (A) AZL-CMC treated wound, day 12, arrow shows collagen deposition in the ulcer (score 3), Masson's trichrome 100x. (B) Control wound, day 12, arrow shows sparse collagen deposition in the ulcer (score I), Masson's trichrome 100x. (C) AZL-CMC treated wound, day 12, complete epithelialization (score 3) and matured granulation tissue (score 3) H \& E 400x. (D) Control wound, day 12, ulcer with partial epithelialization (score I), H \& E I00x.

\section{COLLAGEN DEPOSITION}

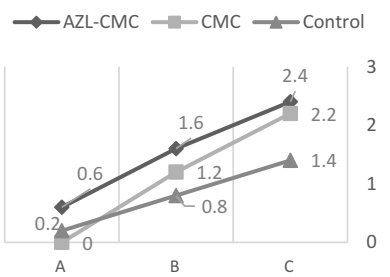

\section{REEPITHELIZATIO}

$\mathbf{N}$

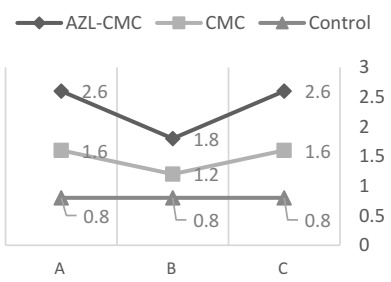

\section{NEOVASCULARIZA TION}

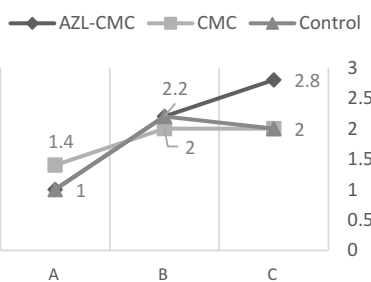

\section{GRANULATION TISSUE}

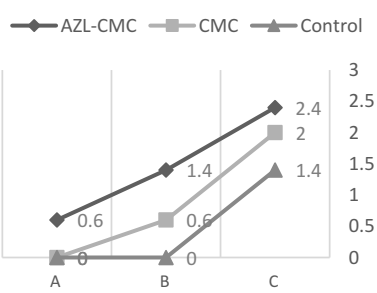

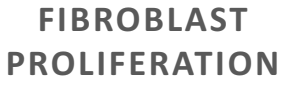

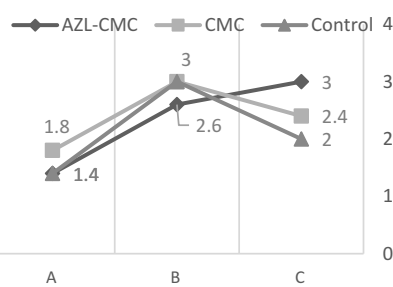

NECROSIS

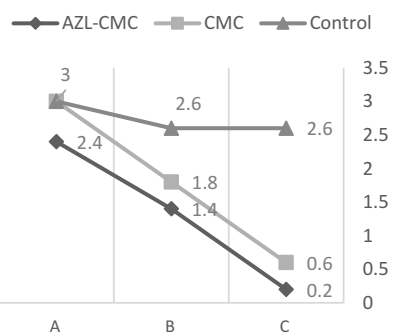

\section{HEMORRHAGE}

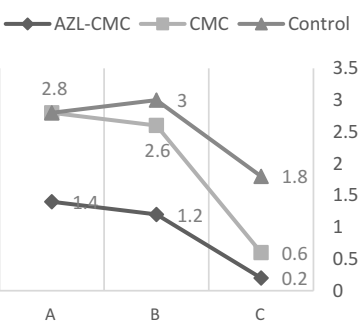

INFLAMMATION

$\longrightarrow$ AZL-CMC - CMC $\longrightarrow$ Control

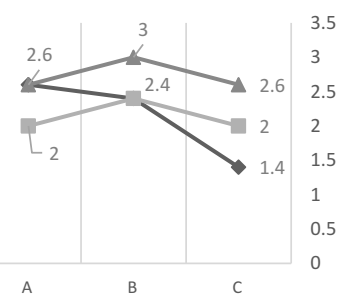

Figure 3 Effect of AZL-CMC gel on wound healing in histopathologic study. 
process in rat models according to stereological parameters. Accordingly, wound closure rate was faster in the verapamil-treated group. The volume density of the collagen bundles, numerical density of the fibroblasts, mean diameter, and volume densities of the vessels in the verapamil group were significantly higher than those in the rats in the control and base groups; this confirmed our achievements that numerical density of the fibroblasts and length density of the vessels in the dermis of the AZL-CMC Gel treated group were significantly higher than those of the control and CMC $2 \%$ treated groups. ${ }^{8}$

Bhaskar et al. in 2005 showed that the two calcium channel blockers, i.e. nifedipine and amlodipine, would enhance the normal healing, as evidenced by increase in the tensile strength of 10-day-old granulation tissues. There was a significant change in neither hydroxyproline level nor collagen or glycosaminoglycan content in the granulation tissue. However, the lysyloxidase level was increased significantly. We did not measure it in our experiment, but in Bhashkar's observation, like our experiment, the tensile strength increased; a also, we showed that the Mean \pm SD of volume density of the collagen bundles was $83.1 \pm 4.46$ in the AZL-CMC treated group and higher compared to the control and CMC 2\%-treated groups, which were both statistically significant. The drugs were also able to overcome steroid depressed wound healing, which makes the same condition as streptozotocin induced diabetes in the rats in our study. ${ }^{29}$

As another article which represents the same results as ours, Hemmati. et al. evaluated the wound-healing activity of calcium channel blocker (amlodipine) and amlodipine with phenytoin combination on excisional cutaneous wound models in rabbits. The results indicated a significant difference between the days needed for complete healing in both treatment groups. Like our study, they showed that wound closure rate in the AZL-CMC Gel treated group was significantly higher in comparison to the control and CMC 2\% treated groups.

Our study had some strengths and limitations. The authors acknowledge that only 3 days were studied for histopathology, and the NO level was not detected in the wounds. This is the first time that we used a combined histology criteria and stereological study for wound healing, so it may help the other wound researchers to do better wound study. This experimental study, regarding the effects of these topical gels, is still on its beginning steps; therefore, we suggest further studies on the effects of other doses of AZL-CMC Gel on the healing process of chronic, burn and diabetic wounds.

\section{Acknowledgment}

The authors would like to thank Dr Nasrin Shokrpour for the editorial assistance in the Research Consulting Center (RCC) of Shiraz University of Medical Science and improvement of the use of English in the manuscript.

\section{Disclosure}

The authors report no conflicts of interest in this work.

\section{References}

1. Patel S, Srivastava S, Singh MR, Singh DJB. Mechanistic insight into diabetic wounds: pathogenesis, molecular targets and treatment strategies to pace wound healing. Pharmacotherapy. 2019;112:108615. doi:10.1016/j.biopha.2019.108615

2. Madmoli M, Dehcheshmeh ZM, Rafi A, Kord Z, Mobarez F, Darabiyan PJMS. The rate of some complications and risk factors of diabetes in diabetic patients: study on cases of 3218 diabetic patients. Med Sci. 2019;23(95):63-68.

3. Sorg H, Tilkorn DJ, Hager S, Hauser J, Mirastschijski UJESR. Skin wound healing: an update on the current knowledge and concepts. Eur Surg Res. 2017;58(1-2):81-94. doi:10.1159/000454919

4. Zgheib C, Hilton SA, Dewberry LC, et al. Use of cerium oxide nanoparticles conjugated with microRNA-146a to correct the diabetic wound healing impairment. J Am Coll Surg. 2019;228(1):107-115. doi:10.1016/j.jamcollsurg.2018.09.017

5. Davis FM, Kimball A, Boniakowski A, Gallagher K. Dysfunctional wound healing in diabetic foot ulcers: new crossroads. Curr diabetes rep. 2018;18(1):2.

6. Edmonds M, Lázaro-Martínez JL, Alfayate-García JM, et al. Sucrose octasulfate dressing versus control dressing in patients with neuroischaemic diabetic foot ulcers (Explorer): an international, multicentre, double-blind, randomised, controlled trial. Lancet Diabetes Endocrinol. 2018;6(3):186-196. doi:10.1016/S2213-8587(17)30438-2

7. Moura LIF, Dias AMA, Carvalho E, de Sousa HC. Recent advances on the development of wound dressings for diabetic foot ulcer treatment-A review. Acta Biomater. 2013;9(7):7093-7114. doi:10.1016/ j.actbio.2013.03.033

8. Ashkani-Esfahani S, Hosseinabadi OK, Moezzi P, et al. Verapamil, a calcium-channel blocker, improves the wound healing process in rats with excisional full-thickness skin wounds based on stereological parameters. Adv Skin Wound Care. 2016;29(8):271-274. doi:10.1097/01. ASW.0000488666.03896.e6

9. Bagheri M, Jahromi BM, Mirkhani H, et al. Azelnidipine, a new calcium channel blocker, promotes skin wound healing in diabetic rats. $J$ Surg Res. 2011;169(1):e101-e107. doi:10.1016/j.jss.2011.02.039

10. Iwai M, Li HS, Chen R, et al. Calcium channel blocker azelnidipine reduces glucose intolerance in diabetic mice via different mechanism than angiotensin receptor blocker olmesartan. J Pharmacol Exp Ther. 2006;319(3):1081-1087. doi:10.1124/jpet.106.108894

11. Mojiri-Forushani H. The role of calcium channel blockers in wound healing. Iran J Basic Med Sci. 2018;21(12):1198-1199. doi:10.22038/ ijbms.2018.29753.7182

12. Lee SE, Lee S. Skin barrier and calcium. Ann Dermatol. 2018;30 (3):265-275.

13. Hemmati AA, Mojiri Forushani H, Mohammad Asgari H. Wound healing potential of topical amlodipine in full thickness wound of rabbit. Jundishapur J Nat Pharm Prod. 2014;9(3):e15638-e. doi:10.5812/ jjnpp.

14. Barret JP, Podmelle F, Lipový B, et al. Accelerated re-epithelialization of partial-thickness skin wounds by a topical betulin gel: results of a randomized phase III clinical trials program. Burns. 2017;43 (6):1284-1294. doi:10.1016/j.burns.2017.03.005 
15. Legon'kova OA, Belova MS. Polymeric materials in treatment of wounds. Polymer Sci Series D. 2015;8(3):235-239. doi:10.1134/ S1995421215030077

16. Saco M, Howe N, Nathoo R, Cherpelis B. Comparing the efficacies of alginate, foam, hydrocolloid, hydrofiber, and hydrogel dressings in the management of diabetic foot ulcers and venous leg ulcers: a systematic review and meta-analysis examining how to dress for success. Dermatol Online J. 2016;22:8.

17. Han G, Ceilley R. Chronic wound healing: a review of current management and treatments. Adv Ther. 2017;34(3):599-610. doi:10.1007/s12325-017-0478-y

18. Gupta A, Kowalczuk M, Heaselgrave W, Britland ST, Martin C, Radecka I. The production and application of hydrogels for wound management: a review. Eur Polym J. 2019;111:134-151. doi:10.1016/j.eurpolymj.2018.12.019

19. Park J-S, An S-J, Jeong S-I, Gwon H-J, Lim Y-M, Nho Y-C. Chestnut honey impregnated carboxymethyl cellulose hydrogel for diabetic ulcer healing. Polymers. 2017;9(7):248.

20. Kilkenny C, Browne WJ, Cuthill IC, Emerson M, Altman DG. Improving bioscience research reporting: the ARRIVE guidelines for reporting animal research. PLoS Biol. 2010;8(6):e1000412. doi:10.1371/journal.pbio.1000412

21. Guillen J. FELASA guidelines and recommendations. J Am Assoc Lab Anim Sci. 2012;51(3):311-321.

22. Abeeleh MA, Ismail ZB, Alzaben KR, et al. Induction of diabetes mellitus in rats using intraperitoneal streptozotocin: a comparison between 2 strains of rats. Eur J Sci Res. 2009;32 (3):398-402.
23. Zekavat O, Amanat A, Karami M, Paydar S, Gramizadeh B, ZareianJahromi M. Wound healing studies using Punica granatum peel: an animal experimental study. Adv Skin Wound Care. 2016;29(5):217225. doi:10.1097/01.ASW.0000481116.16998.55

24. Garcia Y, Breen A, Burugapalli K, Dockery P, Pandit A. Stereological methods to assess tissue response for tissue-engineered scaffolds. Biomaterials. 2007;28(2):175-186. doi:10.1016/j.biomaterials.2006. 08.037

25. Abramov Y, Golden B, Sullivan M, et al. Histologic characterization of vaginal vs. abdominal surgical wound healing in a rabbit model. Wound Repair Regener. 2007;15(1):80-86. doi:10.1111/j.1524475X.2006.00188.x

26. Matsui T, Yamagishi S, Nakamura K, Inoue H. Azelnidipine, a new long-acting calcium-channel blocker, inhibits tumour necrosis factoralpha-induced monocyte chemoattractant protein-1 expression in endothelial cells. J Int Med Res. 2006;34(6):671-675. doi:10.1177/ 147323000603400613

27. Mishra S. Hyper-Glycaemic Effect of Calcium Channel Blockers. International Journal of Medicine Research. 2017;2(2):15-20.

28. Witte MB, Thornton FJ, Tantry U, Barbul A. L-Arginine supplementation enhances diabetic wound healing: involvement of the nitric oxide synthase and arginase pathways. Metab Clin Exp. 2002;51 (10):1269-1273. doi:10.1053/meta.2002.35185

29. Bhaskar HN, Udupa SL, Udupa AL. Effect of nifedipine and amlodipine on dead space wound healing in rats. Indian J Exp Biol. 2005;43(3):294-296.

\section{Publish your work in this journal}

Veterinary Medicine: Research and Reports is an international, peerreviewed, open access journal publishing original research, case reports, editorials, reviews and commentaries on all areas of veterinary medicine. The manuscript management system is completely online and includes a very quick and fair peer-review system. Visit http://www.dovepress.com/testimonials.php to read real quotes from published authors. 